\title{
Evolutionary population synthesis for binary stellar populations
}

\author{
F. Zhang ${ }^{1}$, Z. $\operatorname{Han}^{1}$, L. Li ${ }^{1}$, and J. R. Hurley ${ }^{2}$ \\ 1 National Astronomical Observatories/Yunnan Observatory, Chinese Academy of Sciences, Kunming, 650011, PR China \\ 2 Department of Astrophysics, American Museum of Natural History, Central Park West at 79th Street, New York, \\ NY 10024, USA
}

Received 10 June 2003 / Accepted 8 August 2003

\begin{abstract}
We present integrated colours, integrated spectral energy distributions, and absorption-line indices, for instantaneous burst solar-metallicity binary stellar populations with ages in the range 1-15 Gyr. By comparing the results for populations with and without binary interactions we show that the inclusion of binary interactions makes the appearance of the population substantially bluer - this is the case for each of the quantities we have considered. This effect raises the derived age and metallicity of the population. Therefore it is necessary to consider binary interactions in order to draw accurate conclusions from evolutionary population synthesis work.
\end{abstract}

Key words. star: evolution - binary: evolution - galaxies: star clusters

\section{Introduction}

The majority of current studies of the integrated light of stellar populations using the evolutionary population synthesis (EPS) method - the most direct approach for modelling galaxies have tended to focus solely on the evolution of single stars. However, observations tell us that upwards of $50 \%$ of the stars populating galaxies are expected to be in binary or higher-order multiple systems (Duquennoy \& Mayor 1991; Richichi et al. 1994, for example). Binary evolution, if the component stars are close enough to exchange mass, can drastically alter the evolution path of a star as expected from single star evolution. Binary interactions can also create some important classes of objects, such as blue stragglers (BSs: Pols \& Marinus 1994), and subdwarf B stars (sdBs, also referred as extreme horizontal branch [EHB] stars: Han et al. 2002, 2003). Therefore binary stars have the potential to play an important role in determining the overall appearance of any realistic stellar population and their evolution should be accounted for in population synthesis models. A few EPS studies have previously made advances in this direction (e.g. Pols \& Marinus 1994; Cervino et al. 1997) but only for specialised cases and not to the degree that we present here.

In this letter we assume that all stars are born in binaries and born at the same time, i.e. an instantaneous binary stellar population (BSP). We then model any interactions within these binaries in our EPS models to investigate the effects on the integrated colours, integrated spectral energy distributions

Send offprint requests to: F. Zhang, e-mail: gssephd@public.km.yn.cn
(ISEDs), and absorption feature indices. The outline of the paper is as follows: we describe our EPS models and algorithm in Sect. 2, present our results and some discussion in Sect. 3, and then finally in Sect. 4 we give our conclusions.

\section{Model description}

\subsection{Input physics}

We use the rapid binary star evolution (BSE) algorithm of Hurley et al. (2002) for the binary evolutionary tracks, the empirical and semi-empirical calibrated BaSeL-2.0 model of Lejeune et al. (1997, 1998) for the library of stellar spectra and the empirical fitting functions of Worthey et al. (1994) for the spectral absorption feature indices defined by the Lick Observatory image dissector scanner (referred to as Lick/IDS) system.

The BSE algorithm provides the stellar luminosity $L$, effective temperature $T_{\text {eff }}$, radius $R$, current mass $M$ and the ratio of radius to Roche-lobe radius $R / R_{\mathrm{L}}$ for the component stars, as well as the period $P$, separation $a$ and eccentricity $e$ for a binary system. It is valid for component star masses in the range $0.1 \leq M_{1}, M_{2} \leq 100 M_{\odot}$, metallicity $0.0001 \leq Z \leq 0.03$, and eccentricity $0.0 \leq e<1.0$. The algorithm includes the single star evolution (SSE) package of analytic formulae as presented by Hurley et al. (2000) in its entirety. In fact, for orbits that are wide enough that mass exchange between the component stars does not take place, the evolutionary parameters of the stars are identical to that given by the SSE package. In addition to all aspects of single star evolution, the BSE algorithm models 
processes such as mass transfer, mass accretion, commonenvelope evolution, collisions, supernova kicks, tidal evolution, and all angular momentum loss mechanisms. This is done mostly by using a prescription (or recipe) based approach.

The BaSeL-2.0 stellar spectra library of Lejeune et al. (1997, 1998) provides an extensive and homogeneous grid of low-resolution theoretical flux distributions in the range of 9.1-160000 nm, and synthetic UBVRIJHKLM colours for a large range of stellar parameters: $2000 \leq T_{\text {eff }} / K \leq 50000$, $-1.02 \leq \log g \leq 5.50$, and $+1.0 \leq[\mathrm{Fe} / \mathrm{H}] \leq-5.0$ (where $g$ denotes surface gravity). For this library correction functions have been calculated for each value of the $T_{\text {eff }}$ and for each wavelength in order to yield synthetic UBVRIJHKLM colours matching the empirical colour- $T_{\text {eff }}$ calibrations derived from observations at solar metallicity. Semi-empirical calibrations for non-solar abundances $([\mathrm{Fe} / \mathrm{H}]=-3.5$ to +1.0$)$ have also been established for this version of the library. After correction the most important systematic differences existing between the original model spectra and the observations are eliminated. Furthermore, synthetic $U B V$ and Washington ultraviolet excesses $\delta_{(U-B)}, \delta_{(C-M)}$ and $\delta_{\left(C-T_{1}\right)}$, obtained from the original model spectra of giants and dwarfs, are in excellent agreement with the empirical metal-abundance calibrations.

The empirical fitting functions of Worthey et al. (1994) give Lick/IDS absorption-line indices as a function of $T_{\text {eff }}, \log g$, and metallicity $[\mathrm{Fe} / \mathrm{H}]$. The effective temperature spans a range of $2100 \leq T_{\text {eff }} / K \leq 11000$ and the metallicity is in the range $-1.0 \leq[\mathrm{Fe} / \mathrm{H}] \leq+0.5$. The indices in the Lick system were extracted from the spectra of 460 stars obtained between 1972 and 1984 using the red-sensitive IDS and Cassegrain spectrograph on the $3 \mathrm{~m}$ Shane telescope at Lick Observatory. The spectra cover the range 4000-6400 $\AA$, with a resolution of $\sim 8 \AA$ (Worthey et al. 1994).

\subsection{Model input and Monte Carlo simulations}

For the EPS of a BSP the main input model parameters are: (i) the initial mass function (IMF) of the primaries; (ii) the lower and upper mass cut-offs $M_{1}$ and $M_{\mathrm{u}}$ to the IMF; (iii) the mass-ratio distribution of the binaries; (iv) the distribution of orbital separations; (v) the eccentricity distribution; (vi) the relative age, $\tau$, of the BSP; and (vii) the metallicity $Z$ of the stars.

Using the Monte Carlo method we simulate the real populations by producing $2 \times 10^{5}$ binary systems. For each binary system the initial mass of the primary is chosen from the approximation to the IMF of Miller \& Scalo (1979) as given by Eggleton et al. (1989), i.e.

$$
M_{1}=\frac{0.19 X}{(1-X)^{0.75}+0.032(1-X)^{0.25}},
$$

where $X$ is a random variable uniformly distributed in the range [0,1], and $M_{1}$ is the primary mass in unit of $M_{\odot}$. For each BSP the lower and upper mass cut-offs $M_{1}$ and $M_{\mathrm{u}}$ are taken as $0.1 M_{\odot}$ and $100 M_{\odot}$.

The initial masses of the component stars are assumed to be correlated with the initial mass of the secondary obtained from a uniform mass-ratio distribution (Mazeh et al. 1992; Goldberg \& Mazeh 1994), i.e.

$q=X$

where $q=M_{2} / M_{1}$ is the mass-ratio.

The distribution of separations is taken as constant in $\log a$ for wide binaries and falls off smoothly at close separations:

$\operatorname{an}(a)= \begin{cases}a_{\mathrm{sep}}\left(a / a_{0}\right)^{m}, & a \leq a_{0}, \\ a_{\mathrm{sep}}, & a_{0}<a<a_{1},\end{cases}$

where $a_{\text {sep }} \approx 0.070, a_{0}=10 R_{\odot}, a_{1}=5.75 \times 10^{6} R_{\odot}$ and $m \approx 1.2$. This distribution implies that there are equal numbers of wide binary systems per logarithmic interval, and that approximately $50 \%$ of stellar systems are binary systems with orbital periods less than $100 \mathrm{yr}$ (The fraction is a typical value for the Galaxy, resulting in $\sim 10.1 \%$ of the binaries experiencing Roche lobe overflow during the past $13 \mathrm{Gyr}$, see Han et al. 1995).

We allow eccentric orbits for the binary systems and assume a uniform eccentricity distribution, i.e.

$e=X$.

In our BSPs we assume that all stars are born in binaries in an instantaneous burst of star formation and we vary the age of the BSPs from $1-15$ Gyr. The metallicity is taken as solar $(Z=$ $0.02)$ throughout this work. For the BSE code there are several important input parameters that require mention: the efficiency of common envelope ejection $\alpha_{\mathrm{CE}}$ is taken as 1.0; the Reimers wind mass-loss coefficient $\eta$ is set constant at 0.3 ; and the tidal enhancement parameter $B=0.0$.

\subsection{Algorithm}

Once the initial state of a binary system (the masses of the component stars, $M_{1}$ and $M_{2}$, separation $a$ and eccentricity of the orbit $e$ ) is given, we obtain evolutionary parameters such as luminosity, effective temperature, radius, and mass for the component stars using the BSE algorithm, transform these evolutionary parameters to colours and stellar flux with the BaSeL2.0 stellar spectral model, and obtain absorption feature indices of the Lick/IDS system using the fitting functions of Worthey et al. (1994). By the following Eqs. (5)-(8) we can obtain the integrated colours, monochromatic flux and absorption feature indices for an instantaneous BSP of a particular age and metallicity.

In the following equations, a parameter identified by a capital letter on the left-hand side represents the integrated BSP, while the corresponding parameter in minuscule on the right-hand side is for the $k$ th star. The integrated colour is expressed by

$\left(C_{i}-C_{j}\right)_{\tau, Z}=-2.5 \log \frac{\sum_{k=1}^{n} 10^{-0.4 c_{i}}}{\sum_{k=1}^{n} 10^{-0.4 c_{j}}}$

where $c_{i}$ and $c_{j}$ are the $i$ th and $j$ th magnitude of the $k$ th star.

The integrated monochromatic flux of a BSP is defined as

$F_{\lambda, \tau, Z}=\sum_{k=1}^{n} f_{\lambda}$,

where $f_{\lambda}$ is the SED of the $k$ th star. 


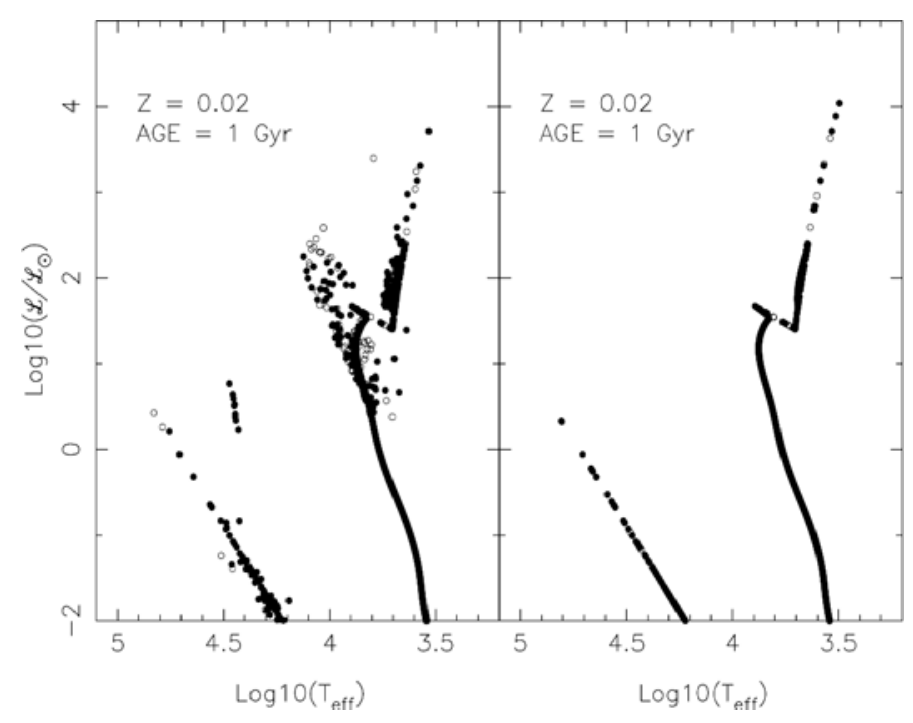

Fig. 1. Theoretical isochrones for solar-metallicity instantaneous burst BSPs at an age of $1 \mathrm{Gyr}$ (solid circles for the primary, open for the secondary). The left panel represents Model A (binary interactions are considered) and the right panel represents Model B (neglecting binary interactions). Blue stragglers and extreme horizontal branch stars are clearly evident in the left panel. For the sake of clarity only $5 \times 2^{4}$ binary systems are included in each panel.

The integrated absorption feature index of the Lick/IDS system is a flux-weighted one. For the $i$ th atomic absorption line, it is expressed in equivalent width ( $W$, in $\AA$ ),

$W_{i, \tau, Z}=\frac{\sum_{k=1}^{n} w_{\mathrm{i}} \cdot f_{i, \mathrm{C} \lambda}}{\sum_{k=1}^{n} f_{i, \mathrm{C} \lambda}}$,

where $w_{\mathrm{i}}$ is the equivalent width of the $i$ th index of the $k$ th star, and $f_{i, \mathrm{C} \lambda}$ is the continuum flux at the midpoint of the $i$ th "feature" passband; and for the $i$ th molecular line, the feature index is expressed in magnitude,

$C_{i, \tau, Z}=-2.5 \log \frac{\sum_{k=1}^{n} 10^{-0.4 c_{i}} \cdot f_{i, \mathrm{C} \lambda}}{\sum_{k=1}^{n} f_{i, \mathrm{C} \lambda}}$,

where $c_{i}$ is the magnitude of the $i$ th index of the $k$ th star (as in Eq. (5)).

\section{Results and discussion}

We have constructed two distinct BSPs in order to investigate the effects of binary interactions on the integrated colours, ISEDs and Lick/IDS absorption feature indices for instantaneous burst solar-metallicity BSPs with ages in the range $1 \leq$ $(\tau /$ Gyr $) \leq 15$ : Model A includes binary interactions by utilising the BSE algorithm whereas Model B neglects all binary interactions, i.e. the component stars are evolved as if in isolation according to the SSE algorithm.

In Fig. 1 we show the theoretical isochrones for Models A and B at an age of $\tau=1 \mathrm{Gyr}$. As expected, we see clearly that the distribution of stars in the Hertzsprung-Russell diagram for Model A is significantly different from that of Model B: (i) the distribution of the stars is more dispersed for Model A in comparison to Model B; and (ii) BSs and EHB stars on the helium main-sequence are produced by Model A only. These differences in the distribution of stars are responsible for differences in the appearance of EPS models with and without binaries. As for old BSPs (such as $\tau=13 \mathrm{Gyr}$ ) the number of BSs in Model A is relatively smaller, and no stars exist on the helium main-sequence. Hurley et al. (2001) compared their theoretical colour-magnitude diagram (CMD) with that of M 67, it shows that our treatment of binaries is realistic.

In Fig. 2 we give a comparison of the integrated $U-B$, $B-V, V-R$ and $V-I$ colours for Models $\mathrm{A}$ and $\mathrm{B}$. We see that the colours for Model A are bluer than those for Model B in all instances, and at some ages the discrepancies become so large, for example the difference in $B-V$ reaches $\sim 0.07$ at $\tau=3 \mathrm{Gyr}$, that we cannot neglect the effects of binary interactions in EPS models.

In Fig. 3 we give the ISEDs over a wide wavelength range, $2.7 \leq \log (\lambda / \AA) \leq 5.2$, for Model A and Model B at ages of $1 \mathrm{Gyr}$ and $13 \mathrm{Gyr}$. The flux is expressed in magnitude and is normalized to zero at $2.2 \mu \mathrm{m}$. Figure 3 shows a significant disagreement of the ISED in the far-ultraviolet region $(3.0<\log (\lambda / \AA)<3.2)$ for $\tau=1$ Gyr BSPs, in that the ISED for Model $\mathrm{A}$ is as much as $\sim 5 \mathrm{mag}$ greater than that for Model B. The main reason for this discrepancy is the existence of the hot BSs in Model A which dominate the ISED in this region. Also Fig. 3 shows that the ISED for Model A exhibits bluer continuum than that for Model B in the visible and infrared regions for $\tau=1 \mathrm{Gyr}$ BSPs - this discrepancy is caused not only by the existence of the hot BSs in Model A but also by the presence of giants whose colours have been altered by binary interaction. For old BSPs (for example $\tau=13 \mathrm{Gyr}$ ) the ISED of Model A is distinguished from that of Model B only in the wavelength range $\log (\lambda / \AA)<3.4$ : it is bluer than that for Model B by no more than $\sim 2$ mag.

In Fig. 4 we show the evolution of two age-sensitive Lick/IDS spectral absorption feature indices, G4300 and $\mathrm{H}_{\beta}$, and two metallicity-sensitive indices, Fe5015 and $\mathrm{Mg}_{2}$, for Models A and B. Each of these indices are bluer for Model A in comparison to Model $\mathrm{B}$ : the $\mathrm{H}_{\beta}$ indice for Model $\mathrm{A}$ is greater by $\sim 0.34 \AA$ at 3 Gyr.

From all the above comparisons, we can draw the conclusion that binary interactions make the integrated colours, ISEDs and Lick/IDS absorption feature indices bluer. As each of these quantities tend to become redder with increasing age and metallicity it follows that the inclusion of binary interactions acts to raise the derived age and metallicity of a particular population. The degree of this increase depends on the method, by which one obtains the age and metallicity.

We also present the differences in the integrated colours and the derived ages between Model A and B in Table 1, and the Lick/IDS indices in Table 2. At early and intermediate ages $(\tau<10 \mathrm{Gyr})$ the differences in the derived ages $(\Delta \mathrm{Age})$ reach the maximum because the number of BSs peaks at $\sim 5 \mathrm{Gyr}$, and at late ages $(\tau<10 \mathrm{Gyr})$ the larger differences in the derived ages result from the facts (1) the colours and Lick/IDS indices increase relatively lowly with age, and (2) Monte Carlo simulations produce some fluctuations in colours and indices, which will be improved in future studies. 

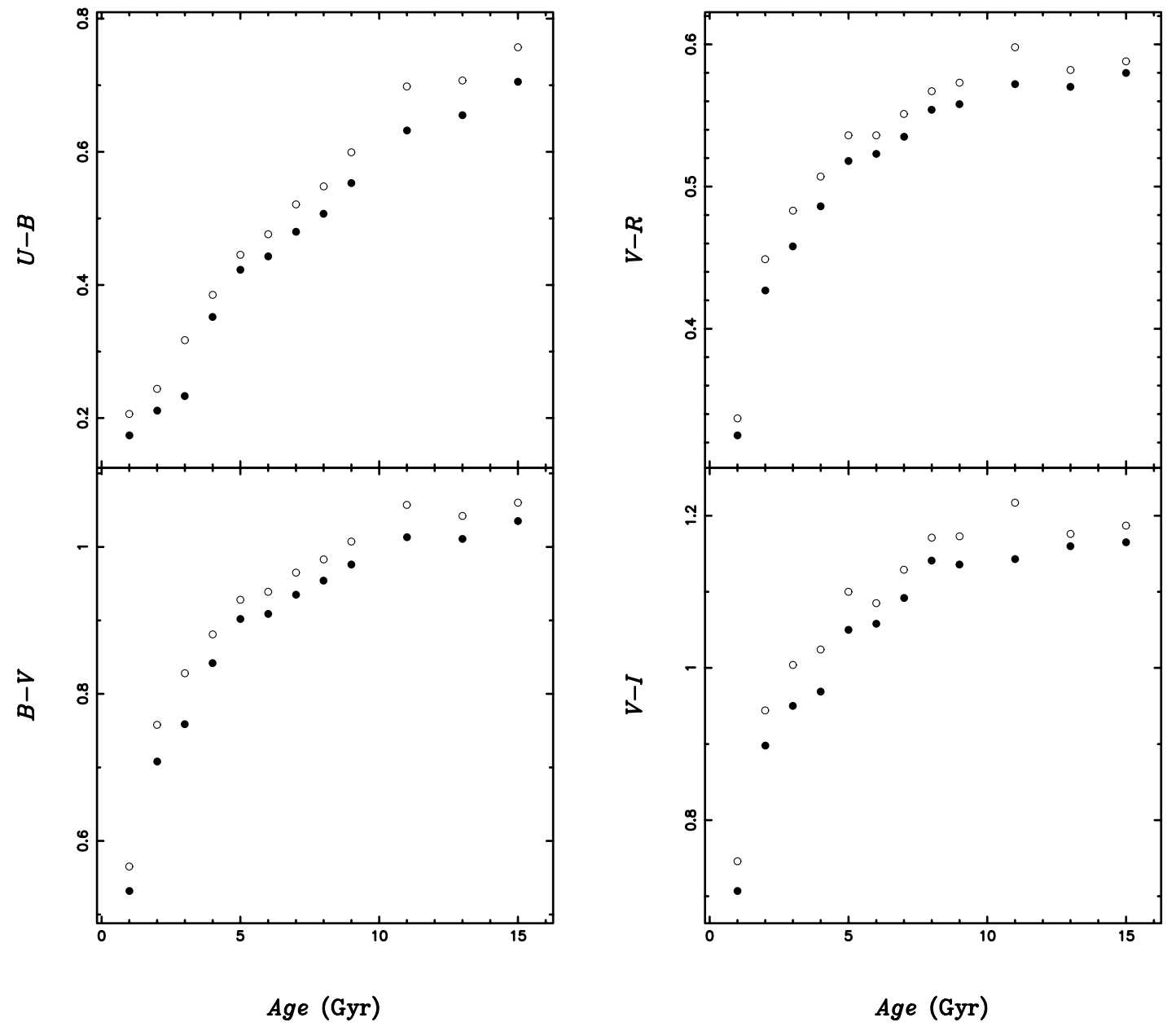

Fig. 2. Integrated colours as a function of age for solar-metallicity instantaneous burst BSPs with (solid) and without (open) binary interactions.

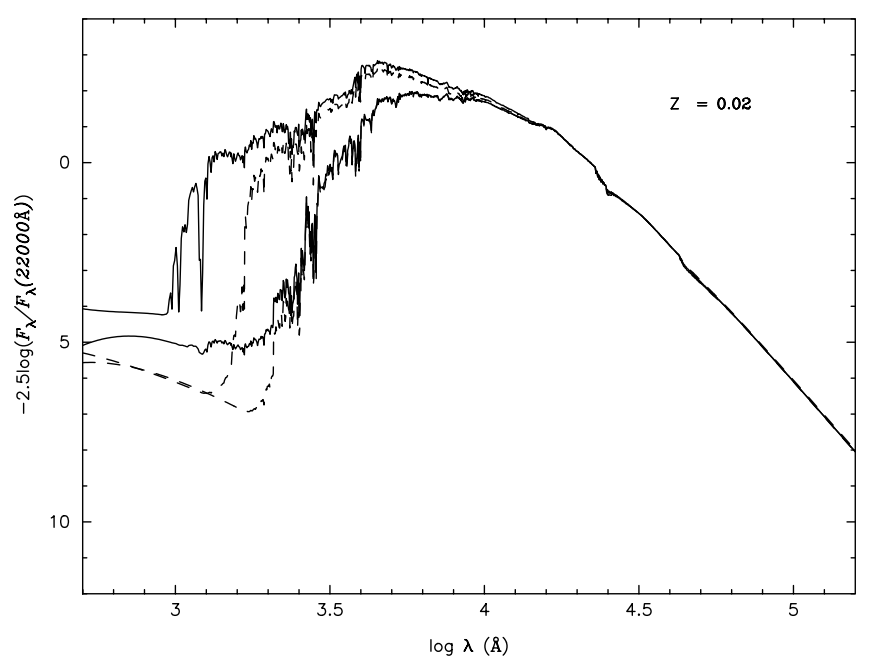

Fig. 3. The integrated spectral energy distributions for solarmetallicity instantaneous burst BSPs with (full line) and without (dashed line) binary interactions at ages of $1 \mathrm{Gyr}$ and $13 \mathrm{Gyr}$ (upper lines and lower lines, respectively). The flux is expressed in magnitude and is normalized to zero at $2.2 \mu \mathrm{m}$.

\section{Summary and conclusions}

We have simulated realistic stellar populations composed of $100 \%$ binaries by producing $2 \times 10^{5}$ binary systems using a Monte Carlo technique. We computed the integrated colours, ISEDs and Lick/IDS absorption feature indices for these instantaneous burst BSPs with and without binary interactions. In comparison we find that modelling binary evolution, and the additional classes of stars that this produces, leads to bluer integrated colours, ISEDs and Lick/IDS absorption feature indices, and therefore makes the derived age and metallicity raise. In this letter we have only considered the effects of binaries for solar metallicity BSPs - more detailed studies will be given later.

Acknowledgements. We acknowledge the generous support provided by the Chinese Natural Science Foundation (Grant No. 19925312, 10073009, 10273020 \& 10303006), Yunnan Natural Science Foundation (Grant No. 2002A0020Q) and by the 973 scheme (NKBRSF G1999075406). We are deeply indebted to Dr. Lejeune for making his BaSeL-2.0 model available to us. We thank an anonymous referee for his/her useful suggestions. 
Table 1. The differences in the integrated colours and the derived ages between model A and B.

\begin{tabular}{ccccccccc}
\hline \hline $\begin{array}{c}\text { Age } \\
(\mathrm{Gyr})\end{array}$ & $\begin{array}{c}\Delta(U-B) \\
(\mathrm{mag})\end{array}$ & $\begin{array}{c}\Delta \mathrm{Age}_{(U-B)} \\
(\mathrm{Gyr})\end{array}$ & $\begin{array}{c}\Delta(B-V) \\
(\mathrm{mag})\end{array}$ & $\begin{array}{c}\Delta \mathrm{Age}_{(B-V)} \\
(\mathrm{Gyr})\end{array}$ & $\begin{array}{c}\Delta(V-R) \\
(\mathrm{mag})\end{array}$ & $\begin{array}{c}\Delta \mathrm{Age}_{(V-R)} \\
(\mathrm{Gyr})\end{array}$ & $\begin{array}{c}\Delta(V-I) \\
(\mathrm{mag})\end{array}$ & $\begin{array}{c}\Delta \mathrm{Age}_{(V-I)} \\
(\mathrm{Gyr})\end{array}$ \\
\hline 1.0 & -0.032 & 0.865 & -0.033 & 0.187 & -0.012 & 0.118 & -0.039 & 0.204 \\
2.0 & -0.033 & 1.500 & -0.050 & 0.980 & -0.022 & 0.710 & -0.046 & 0.885 \\
3.0 & -0.084 & 0.706 & -0.069 & 0.831 & -0.025 & 0.893 & -0.054 & 2.842 \\
4.0 & -0.033 & 0.465 & -0.039 & 0.650 & -0.021 & 0.656 & -0.055 & 0.679 \\
5.0 & -0.022 & 1.100 & -0.026 & 3.714 & -0.018 & 3.600 & -0.050 & 6.250 \\
6.0 & -0.033 & 0.892 & -0.030 & 1.154 & -0.013 & 1.083 & -0.027 & 0.794 \\
7.0 & -0.041 & 1.519 & -0.030 & 1.579 & -0.016 & 0.842 & -0.037 & 0.755 \\
8.0 & -0.041 & 1.449 & -0.029 & 2.506 & -0.013 & 3.500 & -0.030 & 8.750 \\
9.0 & -0.046 & 1.626 & -0.031 & 2.679 & -0.015 & 4.038 & -0.037 & 7.792 \\
11.0 & -0.066 & 2.333 & -0.044 & 3.802 & -0.026 & 7.000 & -0.074 & 4.583 \\
13.0 & -0.052 & 1.838 & -0.031 & 2.679 & -0.012 & 3.231 & -0.016 & 4.667 \\
15.0 & -0.052 & 2.849 & -0.025 & 4.545 & -0.008 & 4.000 & -0.022 & 4.000 \\
\hline
\end{tabular}

Table 2. The difference in Lick/IDS indices and the derived ages between model A and B.

\begin{tabular}{ccccccccc}
\hline \hline $\begin{array}{c}\text { Age } \\
(\mathrm{Gyr})\end{array}$ & $\begin{array}{c}\Delta \mathrm{G} 4300 \\
(\AA)\end{array}$ & $\begin{array}{c}\Delta \mathrm{Age}_{\mathrm{G} 4300} \\
(\mathrm{Gyr})\end{array}$ & $\begin{array}{c}\Delta \mathrm{H}_{\beta} \\
(\AA)\end{array}$ & $\begin{array}{c}\Delta \mathrm{Age}_{\mathrm{H}_{\beta}} \\
(\mathrm{Gyr})\end{array}$ & $\begin{array}{c}\Delta \mathrm{Fe} 5015 \\
(\AA)\end{array}$ & $\begin{array}{c}\Delta \mathrm{Age}_{\mathrm{Fe} 5015} \\
(\mathrm{Gyr})\end{array}$ & $\begin{array}{c}\Delta \mathrm{Mg}_{2} \\
(\mathrm{mag})\end{array}$ & $\begin{array}{c}\Delta \mathrm{Age}_{\mathrm{Mg}_{2}} \\
(\mathrm{Gyr})\end{array}$ \\
\hline 1.0 & -0.245 & 0.121 & 0.139 & 0.090 & -0.187 & 0.222 & -0.004 & 0.096 \\
2.0 & -0.449 & 1.121 & 0.252 & 0.778 & -0.232 & 1.116 & -0.010 & 0.498 \\
3.0 & -0.732 & 0.874 & 0.341 & 0.802 & -0.313 & 1.049 & -0.013 & 0.687 \\
4.0 & -0.324 & 0.797 & 0.168 & 0.852 & -0.197 & 0.654 & -0.011 & 0.722 \\
5.0 & -0.234 & 0.944 & 0.122 & 0.936 & -0.154 & 8.067 & -0.012 & 1.219 \\
6.0 & -0.205 & 0.866 & 0.107 & 0.964 & -0.140 & 1.082 & -0.008 & 0.925 \\
7.0 & -0.162 & 1.652 & 0.084 & 1.231 & -0.165 & 1.681 & -0.008 & 0.709 \\
8.0 & -0.172 & 0.818 & 0.072 & 0.690 & -0.176 & 3.806 & -0.007 & 1.219 \\
9.0 & -0.130 & 1.047 & 0.057 & 0.766 & -0.185 & 3.296 & -0.007 & 1.238 \\
11.0 & -0.134 & 6.781 & 0.042 & 5.954 & -0.328 & 15.570 & -0.010 & 4.703 \\
13.0 & -0.179 & 2.612 & 0.088 & 1.775 & -0.119 & 4.646 & -0.008 & 2.033 \\
15.0 & -0.149 & 2.164 & 0.055 & 1.102 & -0.148 & 5.798 & -0.005 & 1.315 \\
\hline
\end{tabular}
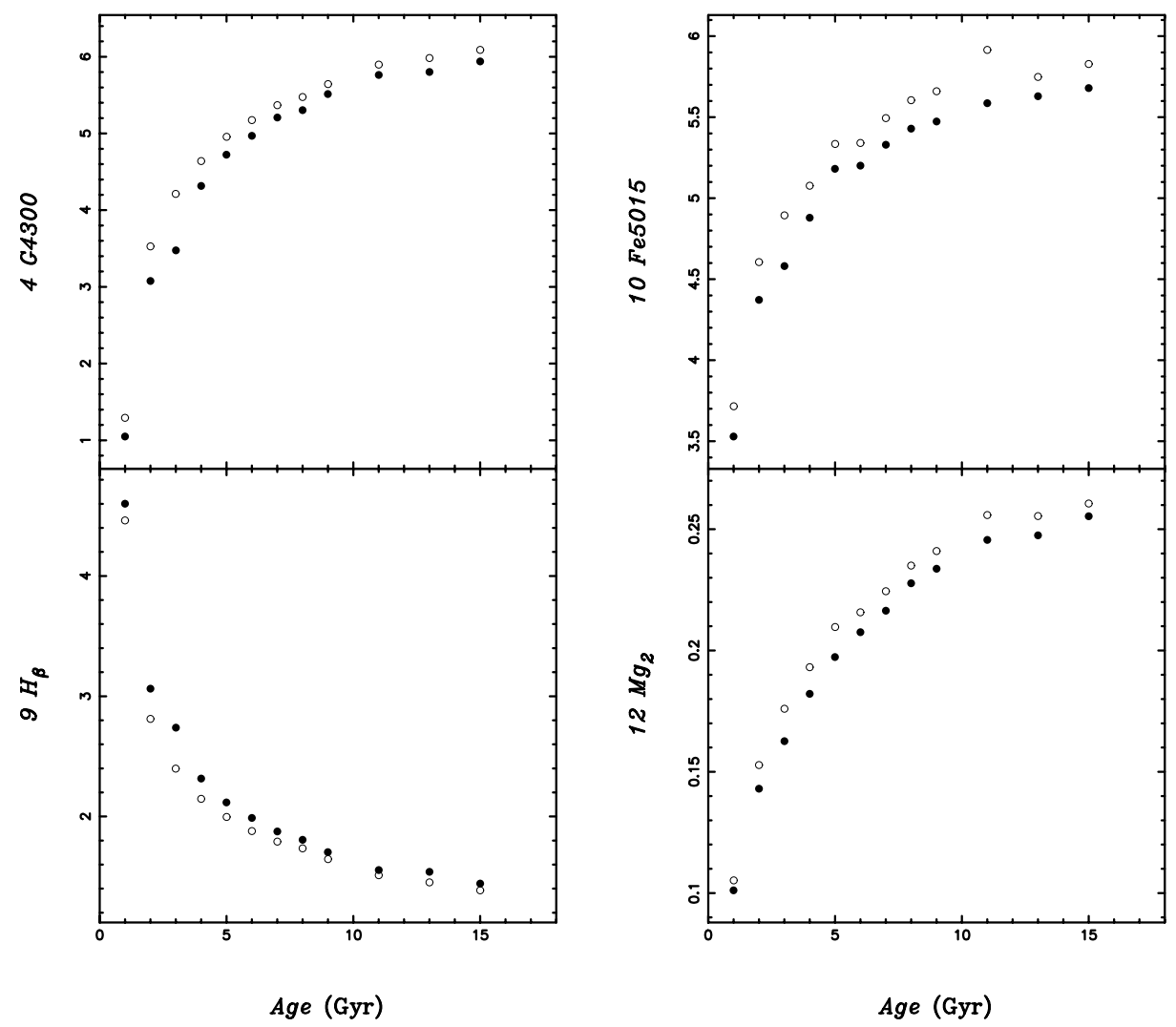

Fig. 4. Evolution of absorption indices in the Lick/IDS system for solar-metallicity instantaneous burst BSPs with (solid) and without (open) binary interactions. 


\section{References}

Cerviño, M., Mas-Hesse, J.-M., \& Kunth, D. 1997, in Rev. Mex. Astron. Astrofis. Conf. Ser., 6, 188

Duquennoy, A., \& Mayor, M. 1991, A\&A, 248, 485

Eggleton, P. P., Fitchett, M. J., \& Tout, C. A. 1989, ApJ, 347, 998

Goldberg, D., \& Mazeh, T. 1994, A\&A, 282, 801

Han, Z., Podsiadlowski, Ph., \& Eggletion, P. P. 1995, MNRAS, 272, 800

Han, Z., Podsiadlowski, Ph., Maxted, P. F. L., Marsh, T. R., \& Ivanova, N. 2002, MNRAS, 336, 449

Han, Z., Podsiadlowski, Ph., Maxted, P. F. L., \& Marsh, T. R. 2003, MNRAS, 341, 669

Hurley, J. R., Pols, O. R., \& Tout, C. A. 2000, MNRAS, 315, 543
Hurley, J. R., Tout, C. A., Aarseth, S. J., \& Pols, O. R. 2001, MNRAS, 323,630

Hurley, J. R., Tout, C. A., \& Pols, O. R. 2002, MNRAS, 329, 897

Lejeune, T., Cuisinier, F., \& Buser, R. 1997, A\&AS, 125, 229

Lejeune, T., Cuisinier, F., \& Buser, R. 1998, A\&AS, 130, 65

Mazeh, T., Goldberg, D., Duquennoy, A., \& Mayor, M. 1992, ApJ, 401,265

Miller, G. E., \& Scalo, J. M. 1979, ApJS, 41, 513

Pols, O. R., \& Marinus, M. 1994, A\&A, 288, 475

Worthey, G., Faber, S. M., Gonzalez, J. J., \& Burstein, D. 1994, ApJS, 94, 687

Richichi, A., Leinart, Ch., Jameson, R., \& Zinnecker, H. 1994, A\&A, 287,145 